

www.elsevier.com/locate/nima

\title{
A new method for pulse analysis of driftless-gas proportional scintillation counters
}

\author{
P.C.P.S. Simões ${ }^{\mathrm{a}}$, D.S. Covita ${ }^{\mathrm{a}}$, J.F.C.A. Veloso ${ }^{\mathrm{a}}$, J.M.F. dos Santos ${ }^{\mathrm{a}, *}$, \\ R.E. Morgado ${ }^{\mathrm{b}}$

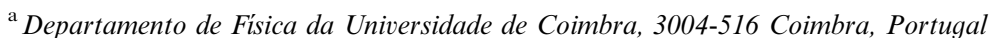 \\ ${ }^{\mathrm{b}}$ Los Alamos National Laboratory, Los Alamos, NM, 87545, USA
}

\begin{abstract}
A new method for pulse analysis of driftless-gas proportional scintillation counters (GPSCs) is presented. With this method the requirement for additional analog or digital signal time-analysis and pulse-amplitude correction currently used is eliminated. In contrast to conventional- and driftless-GPSCs that have always relied on long shaping-time constants (several $\mu \mathrm{s})$, the use of very short linear amplifier shaping-time constants $(\sim 50 \mathrm{~ns})$ enables pulse shapes to closely represent the scintillation light-pulse time-profile. Since the number of detected photons in the photosensor increases continuously with depth due to the increase in the solid angle subtended by the photosensor, a maximum is achieved when the primary electron cloud is closest to the anode. This maximum depends only on the number of primary electrons in that cloud, regardless of where the X-ray absorption took place, and is proportional to the X-ray energy.
\end{abstract}

(C) 2003 Elsevier Science B.V. All rights reserved.

PACS: $07.85 . \mathrm{Nc} ; 29.40 .-\mathrm{n} ; 29.40 . \mathrm{Cs}$

Keywords: Xenon gaseous detectors; Gas proportional scintillation counters; Driftless; Pulse analysis

\section{Introduction}

Xenon-filled gas proportional scintillation counters (GPSCs) have been developed for X-ray spectrometry in astrophysics and high-energy physics $[1,2]$. Their ruggedness, room-temperature operation and large detection area capability are important advantages in many applications.

In conventional GPSCs, X-rays interact primarily in a drift/absorption region with an electric field

*Corresponding author. Tel.: + 351-239-410667; fax: + 351239-829158.

E-mail address: jmf@gian.fis.uc.pt (J.M.F. dos Santos). chosen below the scintillation threshold. The resulting primary electrons drift into a well-defined scintillation region where the electric field is high enough for the drifting electrons to excite but not ionise the gas atoms, producing VUV scintillation. This way, the total amount of light will be independent of the X-ray interaction position.

However, the interaction of $\mathrm{X}$-rays in a region with a weak electric field is disadvantageous for soft X-rays with low penetration depths in the detector, due to the partial loss of primary electrons to the detector radiation window, owing to diffusion. This results in the distortion of pulse-height distributions that depart from the 
gaussian shape resulting in low-energy tails that extend towards very low amplitudes $[3,4]$. Other degrading effects that might be present in the weak electric field of conventional GPSCs with a drift region include primary electron losses to the lowvoltage grid that separates the two regions, losses due to lateral diffusion of the primary electron cloud and losses to impurities due to the higher number of inelastic collisions, for the whole energy range [5].

In driftless GPSCs the drift region is eliminated and X-rays are absorbed directly in the scintillation region, reducing or eliminating the referred degrading effects [5-10]. However, the total amount of light produced as a consequence of an $\mathrm{X}$-ray interaction will depend on the distance traversed by the primary electron cloud in the scintillation region, and, hence, on the depth where this interaction occurred. Since the lightpulse duration is proportional to this distance, the timing analysis of driftless-GPSC pulses allows pulse-amplitude correction to restore the proportionality between the corrected pulse and the corresponding X-ray photon energy.

To implement the required timing-analysis and amplitude correction, an array of analog electronics [7] or costly digitisers with specific software for digital pulse processing [6,8] are needed. This analysis is unnecessary for spectrometry of X-rays with energies below $\sim 2 \mathrm{keV}$, with absorption lengths much smaller than the scintillation region depth, since the effect of the different X-ray penetration depths becomes negligible [7,9]. However, if higher energy X-rays are present, the low energy peaks will be superimposed on a high-level background. Nevertheless, the applicability of a driftless GPSC extends to higher energy ranges [7-10]. Thus, driftless GPSCs are generally used combined with pulse time-analysis and amplitude correction.

In this work we present a new method for driftless-GPSC pulse-analysis using only standard preamplification and amplification electronics, eliminating the need for analog or digital signal time-analysis and pulse-amplitude correction with only a small energy-dependent degradation in the detector performance. This enables trade-offs in designing X-ray spectrometers that do not require the performance and complexity of a fully compensated system.

\section{Method}

In Fig. 1 we depict the schematic diagram of the driftless GPSC used in this work. It has a $10-\mathrm{cm}$ diameter $\times 2.5$-cm deep scintillation region and is filled with xenon at 800 Torr $\left(\sim 1.05 \times 10^{5} \mathrm{~Pa}\right)$, continuously purified. The upper part of the detector body is made of Macor, which insulates the $8-\mathrm{mm}$ diameter Kapton radiation window and its stainless steel holder. The Kapton, the stainless steel and the Macor are epoxied to each other. The Kapton window is aluminised on the inner side to ensure electrical conductivity. The lower part of the detector is built from stainless steel and is connected to the gas circulation tubing. The bottom of the detector is a Macor disc epoxied to a $51-\mathrm{mm}$ diameter PMT and to the detector wall. A chromium grid $(\sim 100-\mu \mathrm{m}$ line width with $1000-\mu \mathrm{m}$ spacing) is vacuum deposited on the PMT quartz window and connected to the photocathode pin through a continuous chromium film deposited on the lateral surface. The upper and lower parts of the detector are made vacuumtight by compression of an indium gasket. The Kapton window and holder are kept at negative high voltage $(-10 \mathrm{kV})$, while the chromium grid and the PMT photocathode are kept at $0 \mathrm{~V}$. The window holder and the upper Macor piece were designed to ensure a uniform electric field in the scintillation region.

As conventional- and driftless GPSCs have always relied on the measurement of the total

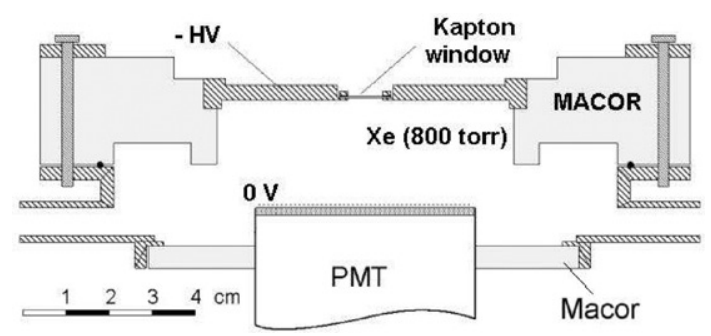

Fig. 1. Schematic of the driftless GPSC used in this work. 
amount of scintillation produced in the scintillation region as a result of the X-ray interaction, long integration and differentiation time constants (several $\mu \mathrm{s}$ ) were needed for pulse shaping, prior to correcting the individual pulse amplitudes. By determining the $\mathrm{X}$-ray signal using the total amount of light collected in the photosensor, the statistical fluctuations associated to the light amplification processes are minimised.

However, the use of very short linear amplifier shaping-time constants $(\sim 50 \mathrm{~ns})$ enables pulse shapes to closely represent the scintillation lightpulse time-profile. Fig. 2 shows typical pulses resulting from $22.1-\mathrm{keV} \mathrm{X}$-ray interactions, when using 50-ns shaping-times. The selected pulses correspond to interactions at different depths and are aligned by the trailing edge. Although the number of scintillation photons produced per electron and per unit of path length is constant at all depths, the number of detected photons increases continuously with depth due to the increase in the solid angle subtended by the photosensor, achieving a maximum when the electron cloud finally reaches the charge-collecting anode.

The maximum is achieved at the same position for every primary electron cloud and its value depends only on the number of primary electrons in that cloud, regardless of where the X-ray absorption took place. This assures proportionality to the X-ray energy [11]. The only exceptions are those absorptions occurring at distances closer to the collection anode than that for which the maximum detected light occurs. The pulses of Fig. 2 show this effect, within the statistical

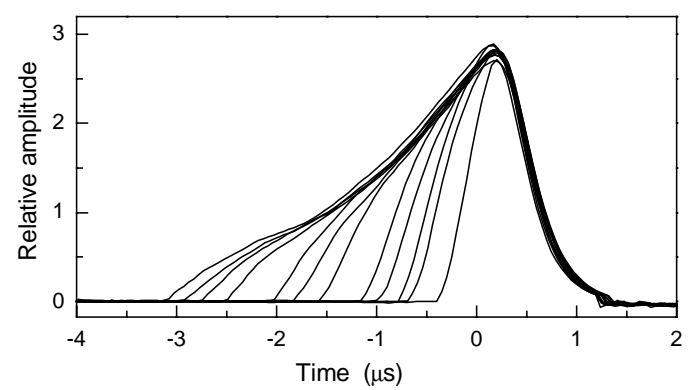

Fig. 2. Typical pulse shapes of the driftless GPSC for 22.1-keV X-ray interactions at different depths. fluctuations: the pulse-height and the trailing edges have the same shape since all the primary electron clouds have the same number of electrons and roughly the same dimensions upon reaching the chromium grid.

Since the amount of scintillation light used to determine the X-ray pulse-height is less than when the total area under the pulse profile is considered, the corresponding statistical fluctuations are somewhat higher. Nevertheless, good performance for many applications can be achieved by pulse-height analysis using very short shaping times, without further processing and compensation, extending the use of simple inexpensive driftless GPSCs based X-ray spectrometers to the high-energy range.

\section{Experimental results and discussion}

In Fig. 3 we depict the pulse-height distributions obtained with the driftless GPSC for ${ }^{55} \mathrm{Fe},{ }^{109} \mathrm{Cd}$ and Dy X-rays for three different conditions: (a) with full digital pulse-shape analysis and amplitude correction, (b) with short shaping-time constants and no additional processing and, for comparison (in Figs. $3 \mathrm{a}$ and b), (c) with long shaping time constants and no additional processing. The $6.4-\mathrm{keV} \mathrm{K}_{\beta}$-line of the ${ }^{55} \mathrm{Fe}$ radioactive source was filtered by a chromium foil. While ${ }^{55} \mathrm{Fe}$ and ${ }^{109} \mathrm{Cd} \mathrm{X}$-rays were $2-\mathrm{mm}$ collimated, the fluorescence of the Dy-target irradiates the entire detector window.

Best detector response is obtained when applying digital pulse processing techniques, achieving energy resolutions (FWHM) of $7.8 \%, 4.5 \%$ and $4.6 \%$ for $5.9,22.1$ and $45.7-\mathrm{keV}$ X-rays, respectively. Digital pulse processing allows not only time-analysis and amplitude correction but also pulse rise-time discrimination. In this way, the background level in the low energy tail of the peaks is reduced.

Nevertheless, the performance achieved with the present technique is not significantly degraded compared to the full-compensation technique. While for $5.9-\mathrm{keV} \mathrm{X}$-rays no differences were found, for $22.1-\mathrm{keV} \mathrm{X}$-rays the energy resolution degrades to $4.8 \%$ and the relative background 

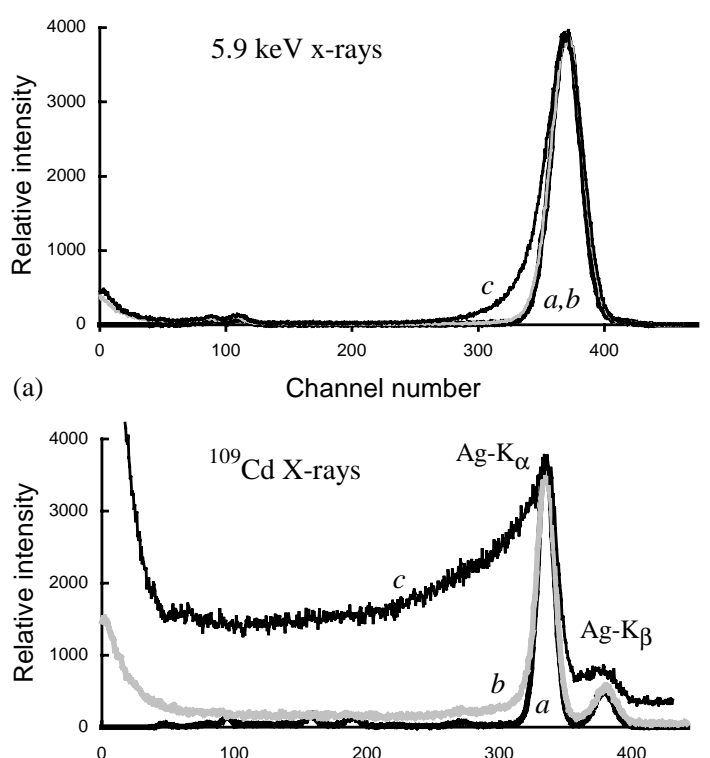

(b)

Channel number

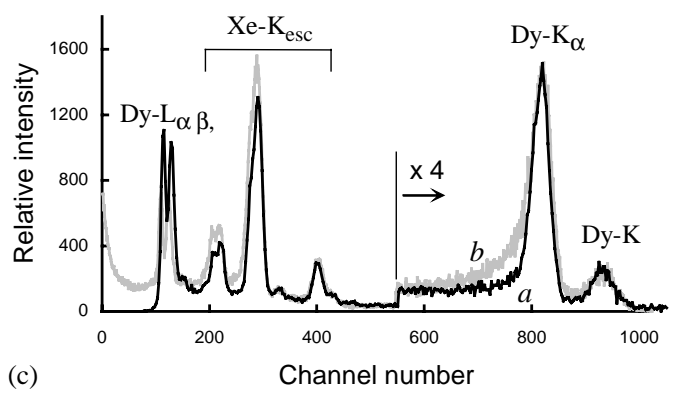

Fig. 3. Pulse-height distributions obtained with the driftless GPSC for ${ }^{55} \mathrm{Fe},{ }^{109} \mathrm{Cd}$ and Dy-fluorescence X-rays: (a) with full digital pulse-shape analysis and compensation; (b) with short shaping-time constants and no additional processing; (c) for long shaping time constants with no additional processing.

level increases by almost a factor of 10, being about $3 \%$ of the peak height. For $42.7-\mathrm{keV}$ $\mathrm{X}$-rays, the energy resolution degrades to $5.5 \%$ and the relative background level increases from $5 \%$ to $8 \%$ of the peak-height. Subtracting the background level from the peaks, assuming it to be a step-function, the skewness of the remaining peak distribution is $0 \%, 1.3 \%$ and $2.4 \%$ of the respective centroid for $5.9,22.1$ and $42.7 \mathrm{keV}$, respectively.

Additionally, pulse amplitudes are reduced by a factor of about 11 . On the other hand, shorter shaping-times result in shorter pulse-duration, allowing higher count-rates.

Initial results obtained with this method with a 1-cm deep driftless GPSC [11] are satisfactory only for X-ray energies below $\sim 8 \mathrm{keV}$. Above that energy, the small GPSC depth results in a significant fraction of X-ray interactions in the region near the anode. Increasing the thickness to $2.5 \mathrm{~cm}$ the performance improves significantly and good results are obtained even for energies in the range of $40-50 \mathrm{keV}$. It is expected that improved performance can be obtained with a larger depth and for a larger energy range.

\section{Conclusions}

The use of very short shaping-time constants $(\sim 50 \mathrm{~ns})$ in the linear amplifier enables X-ray spectrometry of driftless GPSCs to be extended to higher energies, without pulse time-analysis and amplitude correction. Additionally, higher countrates are allowed. Experimental results show that, for a detector with a depth of $2.5 \mathrm{~cm}$, this simple technique is effective for energies up to about $50 \mathrm{keV}$. Improved performance as well as an extended energy range can be reached with a larger detector depth.

\section{Acknowledgements}

Support from project POCTI/ 43527/99 is acknowledged. Travel support is acknowledged for JMF dos Santos to "Fundação Luso-Americana para o Desenvolvimento" and "Fundação Calouste Gulbenkian", Lisbon, and for JFCA Veloso to FCT and Fundação Calouste Gulbenkian, Lisbon.

\section{References}

[1] V.P. Varvaritsa, et al., Instr. Exp. Tech. 35 (1992) 745.

[2] J.M.F. dos Santos, et al., X-ray Spectrom. 30 (2001) 373.

[3] H. Inoue, et al., Nucl. Instr. and Meth. A 157 (1978) 295.

[4] F.P. Santos, et al., IEEE Trans.. Nucl. Sci. NS-42 (1995) 611.

[5] D.J. Simons, et al., Proc. SPIE. 597 (1985) 190. 
[6] A. Smith, et al., IEEE Trans. Nucl. Sci. NS-34 (1987) 57.

[7] D.G. Simons, et al., Nucl. Instr. and Meth. A 277 (1989) 642.

[8] P.C.P.S. Simões, et al., X-ray Spectrom. 30 (2001) 342.
[9] M. Barbera, et al., Proc. SPIE. 2280 (1994) 154.

[10] M. Badvaz, et al., Nucl. Instr. and Meth. A 345 (1994) 549.

[11] P.C.P.S. Simões, et al., IEEE Trans. Nucl. Sci. NS-49 (2002) 912. 